\title{
Modification of Borrelia burgdorferi to overproduce OspA or VlsE alters its infectious behaviour
}

\author{
Correspondence \\ Fang Ting Liang \\ fliang@vetmed.Isu.edu
}

Received 17 April 2008

Revised 10 July 2008

Accepted 2 September 2008

\author{
Qilong $\mathrm{Xu}$, Kristy McShan and Fang Ting Liang
}

Department of Pathobiological Sciences, Louisiana State University, Baton Rouge, LA 70803, USA

\begin{abstract}
The surface lipoproteins of the Lyme disease spirochaete Borrelia burgdorferi directly interact with tissue microenvironments during mammalian infection, and thus potentially affect various aspects of infection. To investigate the influence of surface antigen synthesis on infectious behaviour, $B$. burgdorferi was modified to constitutively produce the well-characterized surface lipoproteins OspA and invariant VlsE. Although increasing OspA or VlsE production did not significantly affect synthesis of other surface lipoproteins or spirochaetal growth in vitro, overexpressing $v / s E$ resulted in increased osp $A$ but decreased ospC expression, and overexpressing ospA led to decreased ospC and $v / s E$ expression in severe combined immunodeficient (SCID) mice. Increasing the expression of either ospA or $v / s E$ did not alter the $\mathrm{ID}_{50}$, but affected spirochaetal dissemination and significantly reduced tissue spirochaete loads in SCID mice. In immunocompetent mice, increased $v / s E$ expression resulted in quick clearance of infection, while constitutive $\operatorname{osp} A$ expression led to a substantial $I_{50}$ increase and severely impaired dissemination. Furthermore, $B$. burgdorferi with constitutive osp $A$ expression persisted in the skin tissue but was cleared from both heart and joints of chronically infected immunocompetent mice. Taken together, the study indicates that increasing production of OspA or invariant VlsE influences lipoprotein gene expression in the murine host and alters the infectious behaviour of $B$. burgdorferi.
\end{abstract}

\section{INTRODUCTION}

The Lyme disease spirochaete Borrelia burgdorferi vigorously modifies its surface antigen expression to adapt to various environments during the enzootic cycle. It abundantly expresses outer surface proteins (Osps) A and B in the unfed tick (de Silva et al., 1996; Ohnishi et al., 2001; Schwan et al., 1995; Schwan \& Piesman, 2000), consistent with a critical role for these lipoproteins in spirochaetal persistence in the vector (Neelakanta et al., 2007; Yang et al., 2004). A fresh blood meal downregulates $\mathrm{OspA} / \mathrm{B}$ and upregulates OspC and others, a process that prepares B. burgdorferi for infection of mammals (Fingerle et al., 2007; Grimm et al., 2004; Pal et al., 2004; Stewart et al., 2006). The downregulation of OspA and OspB during mammalian infection is critical for the maintenance of the enzootic cycle because their expression would ultimately

Abbreviations: Osp, outer surface protein; qPCR, quantitative PCR; RTqPCR, reverse-transcription GPCR; SCID mice, severe combined immunodeficient mice.

Supplementary material describing the determination of $\mathrm{ID}_{50}$ values, and two supplementary tables showing the influence of increasing OspA and VIsE expression on dissemination and $I_{50}$ of $B$. burgdorferi in SCID mice, and that constitutive ospA expression severely impairs dissemination and significantly increases $I_{50}$ in immunocompetent mice, are available with the online version of this paper. induce strong humoral responses to effectively block acquisition by the vector (de Silva et al., 1997; Tsao et al., 2001, 2004), regardless of whether OspA or OspB can be effectively targeted by borreliacidal antibodies in mammalian tissues (Strother et al., 2007). B. burgdorferi abundantly expresses $o s p C$ only during early infection when the antigen may be crucial (Grimm et al., 2004; Stewart et al., 2006; Tilly et al., 2006). However, its expression induces a robust humoral response that imposes tremendous pressure on the pathogen (Fung et al., 1994; $\mathrm{Xu}$ et al., 2006). To cause persistent infection, B. burgdorferi must downregulate $\operatorname{ospC}$ as the specific humoral immune response is developing (Liang et al., 2002a, b, 2004b). It is also crucial for B. burgdorferi to downregulate $\operatorname{sp} \mathrm{C}$ after it is acquired by the tick, as OspC antibodies in the blood meal may kill spirochaetes that express the antigen in the vector (Gilmore \& Piesman, 2000 ), leading to discontinuation of the enzootic cycle.

B. burgdorferi also constantly modifies its surface antigenic expression in response to tissue microenvironmental changes, including the development of humoral responses, during the course of mammalian infection (Bykowski et al., 2007; Crother et al., 2004; Gilmore et al., 2007; Liang et al., 2002a, b, 2004b). The pathogen does not actively express VlsE, the surface variable antigen identified in $B$. 
burgdorferi, during early infection (Liang et al., 2004b). After dissemination into the joint, the pathogen upregulates $v l s E$, while maintaining low expression levels in the skin and heart tissues in the absence of humoral immune responses (Liang et al., 2004b). As the specific humoral response develops, B. burgdorferi downregulates OspC and many other surface antigens but dramatically upregulates VlsE and BBF01 (Crother et al., 2004; Liang et al., 2002a, b, $2004 \mathrm{~b}$ ), a process that probably allows the pathogen to more effectively evade the immune system and cause persistent infection.

As an extracellular bacterium, B. burgdorferi abundantly produces outer surface lipoproteins, which are directly involved in the interplay between the pathogen and tissue microenvironments and are likely to influence its infectious behaviour. To investigate the issue, $B$. burgdorferi was modified to overexpress either ospA or an invariant $v l s E$ gene, and then examined for the $\mathrm{ID}_{50}$, dissemination, tissue colonization and persistence in the murine model.

\section{METHODS}

Strains and constructs generated previously and used in the current study. The B. burgdorferi $\mathrm{B} 31$ clone $13 \mathrm{~A}$, the transformants $13 \mathrm{~A} / \mathrm{E} 22 / \mathrm{C}$ and $13 \mathrm{~A} / \mathrm{E} 22 / \mathrm{D}$, and the ospA mutant $\Delta$ ospA were generated previously (Xu et al., 2007a, b, 2008). The constructs pBBE22-osp $A^{\prime}$ and $\mathrm{pBBE} 22-v l s E^{\prime}$ were generated in a previous study (Xu et al., 2008). The features of these clones and constructs are summarized in Table 1.

Generation of transformants. The clones $13 \mathrm{~A}$ and $\triangle$ osp $A$ were

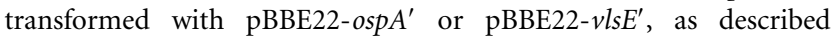
previously ( $\mathrm{Xu}$ et al., 2007a). Transformants were identified and plasmid content was analysed as described previously (Xu et al., 2005). Selected transformants were analysed for OspA, OspC and VlsE expression by immunoblotting probed with a mixture of FlaB, OspA and OspC mAbs, or mouse antisera raised against a recombinant VlsE, as described previously (Xu et al., 2008).

In vitro growth kinetics and serum resistance. Spirochaetes were

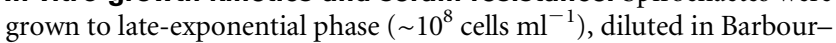
Stoenner-Kelly H (BSK-H) complete medium (Sigma) or BSK-H medium supplemented with mouse sera at the ratio of $1: 1$ (v/v) to a density of $\sim 10^{6}$ cells $\mathrm{ml}^{-1}$ and cultured at $33^{\circ} \mathrm{C}$. Mouse sera were prepared from blood collected from 10 severe combined immunodeficient (SCID) mice. The bacteria grown in BSK-H medium were counted every $24 \mathrm{~h}$ until reaching late-exponential phase. The organisms grown in the mixture of BSK-H and sera were assessed for viability every $12 \mathrm{~h}$ for 3 days under a darkfield microscope.

Infectivity and pathogenicity in SCID mice. BALB/c SCID mice (age 4 to 8 weeks; provided by the LSU Division of Laboratory Animal Medicine) were given one single intradermal/subcutaneous injection of $10^{4}$ spirochaetes. Animals were examined for the development of arthritis at 2-day intervals, starting at 10 days; animals were sacrificed 1 month post-inoculation. Tibiotarsal joint, heart and skin (not from the inoculation site) specimens were used for spirochaete isolation, and DNA and RNA preparation. DNA was quantified for bacterial loads by quantitative PCR (qPCR), as previously described (Xu et al., 2005). RNA was quantified for the mRNA copy numbers of flaB, ospA, ospC and $v l s E$ by reverse-transcription qPCR (RT-qPCR), as described previously (Liang et al., 2004a). The expression levels were presented as ospA, ospC or $v l s E$ mRNA copy numbers per $10000 \mathrm{flaB}$ transcripts.

Infectivity in immunocompetent mice. $\mathrm{BALB} / \mathrm{c}$ mice each received one single intradermal/subcutaneous injection of $10^{5}$ spirochaetes. Some animals were euthanized 1 month post-inoculation; heart, tibiotarsal joint and skin specimens were aseptically collected for spirochaete culture as previously described (Xu et al., 2005), and blood was collected for ELISAs. Other inoculated mice were euthanized 1,2, 3 and 4 weeks post-inoculation; the inoculation site and remote skin, ear, heart and joint specimens were aseptically harvested for spirochaete isolation.

Measurement of anti-OspA and -VIsE humoral immune response. Specific OspA and VlsE antibody end point titres were determined by ELISAs. Ninety-six-well plates (Fisher) were coated with $100 \mu \mathrm{l}$ of $2.0 \mu \mathrm{g} \mathrm{ml}^{-1}$ recombinant OspA or VlsE per well. Sera were twofold serially diluted, starting at $1 / 200$. Five samples drawn from naive $\mathrm{BALB} / \mathrm{c}$ mice were used as a control. The ELISA was performed as previously described (Xu et al., 2006).

Chronic infectivity. Subgroups of five BALB/c mice were given one single intradermal/subcutaneous injection of $10^{5}$ spirochaetes. Mice were euthanized 4 months post-inoculation; heart, tibiotarsal joint and skin specimens were aseptically collected for spirochaete culture, as previously described (Xu et al., 2005).

Table 1. Constructs and clones used in the study

\begin{tabular}{|c|c|c|}
\hline Construct or clone & Description & Source \\
\hline pBBE22-osp $A^{\prime}$ & pBBE22 carrying promoterless ospA fused with $f l a B$ promoter & $\mathrm{Xu}$ et al. (2008) \\
\hline pBBE22-vlsE & pBBE22 carrying promoterless $v l s E$ fused with $f l a B$ promoter & Xu et al. (2008) \\
\hline $13 \mathrm{~A}$ & Cloned from B. burgdorferi $\mathrm{B} 31 \mathrm{~A} 13$ & $\mathrm{Xu}$ et al. (2007a) \\
\hline$\Delta o s p A$ & ospA mutant generated from $13 \mathrm{~A}$ & $\mathrm{Xu}$ et al. (2008) \\
\hline $13 \mathrm{~A} / \mathrm{E} 22 / \mathrm{C}$ & $13 \mathrm{~A}$ transformed with pBBE22 & $\mathrm{Xu}$ et al. (2007b) \\
\hline 13A/E22/D & $13 \mathrm{~A}$ transformed with $\mathrm{pBBE} 22$ & $\mathrm{Xu}$ et al. (2007b) \\
\hline$\Delta o s p A / o s p A^{\prime} / 1$ & osp $A$ mutant expressing osp $A$ driven by $f l a B$ promoter & This study \\
\hline$\Delta o s p A / o s p A^{\prime} / 2$ & osp $A$ mutant expressing osp $A$ driven by flaB promoter & This study \\
\hline $13 \mathrm{~A} / \operatorname{osp} A^{\prime} / 1$ & $13 \mathrm{~A}$ expressing osp $A$ driven by $f l a B$ promoter & This study \\
\hline $13 \mathrm{~A} / \operatorname{osp}^{\prime} / 2$ & $13 \mathrm{~A}$ expressing osp $\mathrm{A}$ driven by flaB promoter & This study \\
\hline $13 \mathrm{~A} / v l s E^{\prime} / 1$ & $13 \mathrm{~A}$ expressing $v l s E$ driven by $f l a B$ promoter & This study \\
\hline $13 \mathrm{~A} / v l s E^{\prime} / 2$ & $13 \mathrm{~A}$ expressing $v l s E$ driven by $f l a B$ promoter & This study \\
\hline
\end{tabular}


Statistical analysis. A one-way analysis of variance (ANOVA) was used, followed by a two-tailed Student's $t$ test to calculate a $P$ value for each pair of treatments. Fisher's exact test was used to analyse data on ear biopsy and frequency of tissue colonization. A $P$ value $\leqslant 0.05$ was considered to be significant.

\section{RESULTS}

\section{Generation of B. burgdorferi with increased OspA or VIsE production}

The constructs pBBE22-osp $A^{\prime}$ and pBBE22- $v l s E^{\prime}$ were electroporated into the 13A spirochaetes. Eleven and 19 transformants were obtained from transformation with each construct. Because clone $13 \mathrm{~A}$ lacks $1 \mathrm{p} 25$, a plasmid that harbours $b b e 22$, a gene encoding a nicotinamidase essential for survival of B. burgdorferi in the mammalian environment (Purser et al., 2003), both constructs were derived from pBBE22 (Xu et al., 2008) and thus carried a copy of bbe22, and should restore the infectivity of clone 13A. Plasmid content analyses identified two clones that received each construct, namely $13 \mathrm{~A} / \operatorname{os} p A^{\prime} / 1,13 \mathrm{~A} / o s p A^{\prime} / 2,13 \mathrm{~A} / v l s E^{\prime} / 1$ and $13 \mathrm{~A} / v l s E^{\prime} / 2$. These clones had identical plasmid content: all lost cp9, lp21 and lp5, in addition to lp25 and lp56. Increased VlsE expression resulting from the introduction of pBBE22- $v l s E^{\prime}$ was easily confirmed by immunoblotting probed with mouse anti-VlsE sera (Fig. 1a). Unfortunately, due to overwhelming expression resulting from the native ospA copy, the potential contribution of pBBE22-osp $A^{\prime}$ to OspA production could not be demonstrated in clone 13A/ osp $A^{\prime} / 1$ or clone $13 \mathrm{~A} / \operatorname{os} p A^{\prime} / 2$ (Fig. 1a). The immunoblot also showed that introduction of the constructs did not influence OspC production.

To examine whether pBBE22-osp $A^{\prime}$ drove ospA expression, the construct was electroporated into $\triangle$ ospA. Twelve transformants were obtained; two clones, namely $\triangle \operatorname{osp} A / o s p A^{\prime} / 1$ and $\Delta o s p A / o s p A^{\prime} / 2$, were selectively analysed by immunoblotting. Both clones abundantly produced OspA antigen (Fig. 1b).

\section{OspA or VIsE overproduction does not affect spirochaete growth or serum resistance in vitro}

The influence of lipoprotein overproduction on spirochaete growth and serum resistance was investigated. The $13 \mathrm{~A} / \mathrm{E} 22 / \mathrm{C}, 13 \mathrm{~A} / \operatorname{osp} A^{\prime} / 1$ and $13 \mathrm{~A} / v l s E^{\prime} / 1$ spirochaetes completed a generation approximately every $7 \mathrm{~h}$ when grown in BSK-H, and all three genotypes survived equally well in BSK-H/sera (data not shown), indicating that overproduction of OspA or VlsE does not influence growth or serum resistance in vitro.

\section{Constitutive ospA expression represses vIsE and ospC expression, and increased vIsE expression enhances ospA but represses ospC expression during infection of SCID mice}

To confirm in vivo increased ospA and $v l s E$ expression, subgroups of five SCID mice were challenged with $10^{4}$
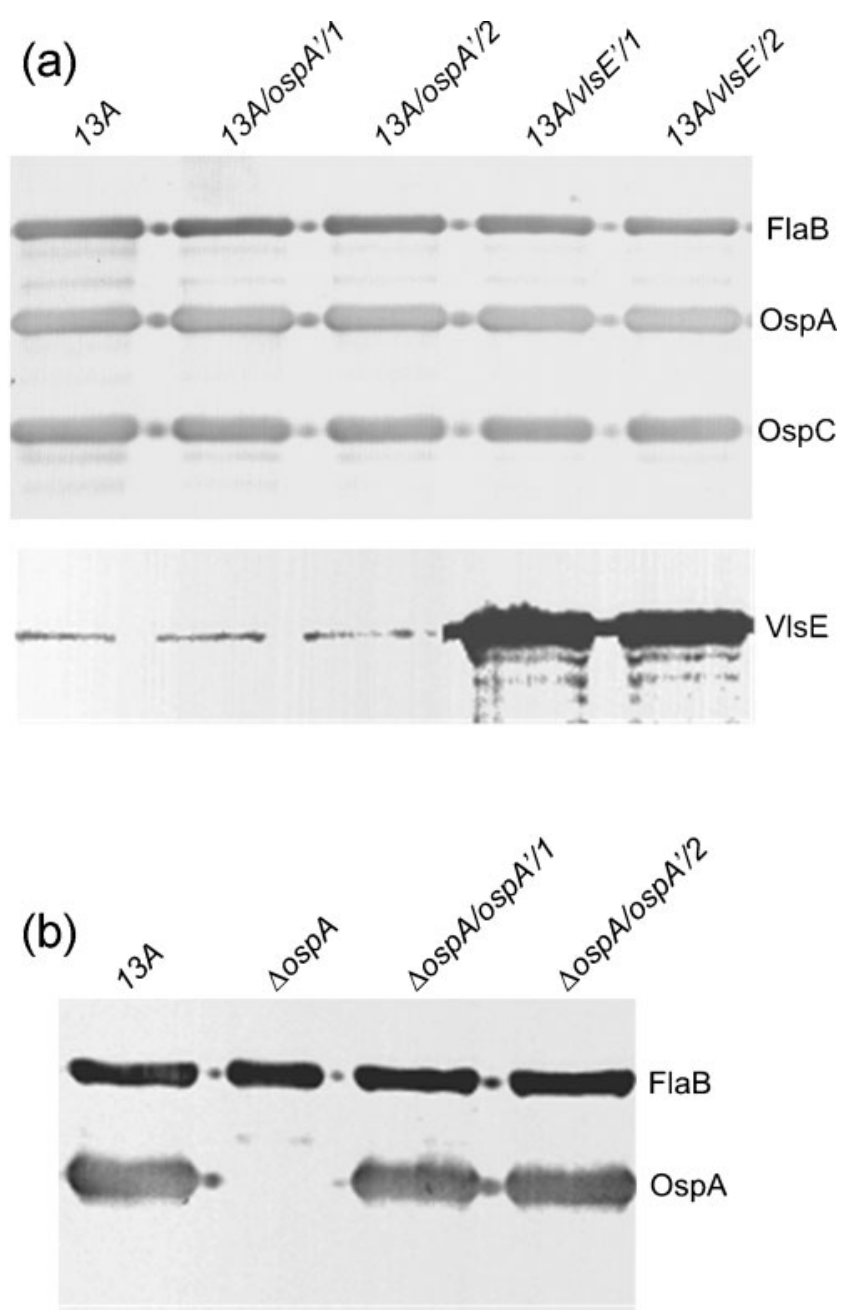

Fig. 1. Confirmation of increased OspA and VlsE expression. The 13A, 13A/ospA'/1, 13A/ospA'/2, 13A/v/sE'/1 and 13A/v/sE'/2 spirochaetes were grown to late-exponential phase and subjected to immunoblot analyses probed with a mixture of FlaB, OspA and OspC mAbs (a, top panel) or mouse anti-VlsE sera (bottom panel). The 13A, $\Delta o s p A / o s p A^{\prime} / 1$ and $\Delta o s p A / o s p A^{\prime} / 2$ spirochaetes were also analysed by immunoblotting probed with a mixture of $\mathrm{FlaB}$ and OspA mAbs (b).

spirochaetes of the clones $13 \mathrm{~A} / \operatorname{os} p A^{\prime} / 1,13 \mathrm{~A} / \operatorname{osp}^{\prime} / 2,13 \mathrm{~A} /$ $v l s E^{\prime} / 1$ or $13 \mathrm{~A} / v l s E^{\prime} / 2$. As a control, an additional 10 mice were inoculated with the clones $13 \mathrm{~A} / \mathrm{E} 22 / \mathrm{C}$ or $13 \mathrm{~A} / \mathrm{E} 22 / \mathrm{D}$. The two clones were previously generated by transforming the 13A spirochaetes with pBBE22 (Xu et al., 2007b). Joint swelling started to develop in all mice at approximately 10 days, and quickly developed to severe arthritis (data not shown), indicating that introduction of pBBE22-osp $A^{\prime}$ or pBBE22-vlsE' did not affect arthritis virulence.

Infected mice were euthanized 1 month post-inoculation; RNA was prepared from heart, joint and skin specimens and assessed for the relative copy numbers of $\operatorname{ss} A, \operatorname{ss} p C$, $v l s E$ and $f l a B$ mRNAs by RT-qPCR. The $13 \mathrm{~A} /$ osp $A^{\prime}$ bacteria accumulated ospA mRNA 3189-, 1034- and 180-fold more 
than the genotype 13A/E22 in heart $\left(P=6.8 \times 10^{-12}\right)$, joint $\left(P=4.4 \times 10^{-12}\right)$ and skin tissues $\left(P=5.1 \times 10^{-15}\right)$, respectively (Fig. 2a). However, they reduced osp $C$ expression 49 and $22 \%$ in heart $\left(P=4.9 \times 10^{-6}\right)$ and skin $(P=0.002)$, respectively, but increased expression 10.3 -fold in joint tissue $\left(P=2.9 \times 10^{-9}\right)$ (Fig. 2b). The genotype 13A/osp $A^{\prime}$ also reduced $v l s E$ mRNA accumulation 4.9-, 5.8- and 2.1fold in heart $\left(P=5.7 \times 10^{-3}\right)$, joint $\left(P=2.7 \times 10^{-10}\right)$ and skin tissues $(P=0.03)$, respectively (Fig. $2 c)$.

Introduction of pBBE22- $v l s E^{\prime}$ increased $v l s E$ expression 10.2-, 2.1- and 16.7-fold over the genotype 13A/E22 in heart $\left(P=3.4 \times 10^{-9}\right)$, joint $\left(P=1.4 \times 10^{-6}\right)$ and skin tissues $\left(P=2.3 \times 10^{-9}\right)$, respectively (Fig. $2 \mathrm{c}$ ). The pBBE22-vlsE' spirochaetes increased ospA expression 62-, 39- and 4.8-fold in heart $\left(P=4.4 \times 10^{-4}\right)$, joint $\left(P=6.0 \times 10^{-5}\right)$ and skin tissues $(P=0.001)$, respectively (Fig. $2 \mathrm{a}$ ), but reduced os $p C$ expression 6.4-, 10.1- and 13.2-fold in heart $\left(P=3.7 \times 10^{-11}\right)$, joint $\left(P=1.6 \times 10^{-5}\right)$ and skin tissues $\left(P=1.2 \times 10^{-13}\right)$, respectively (Fig. 2b).

\section{Increasing expression of either ospA or vIsE shows a tissue-dependent influence on colonization in SCID mice}

DNA was prepared from the heart, joint and skin specimens harvested from the 30 infected mice described above, and quantified for tissue spirochaete burden by $\mathrm{qPCR}$ as an indication of tissue colonization. As shown in
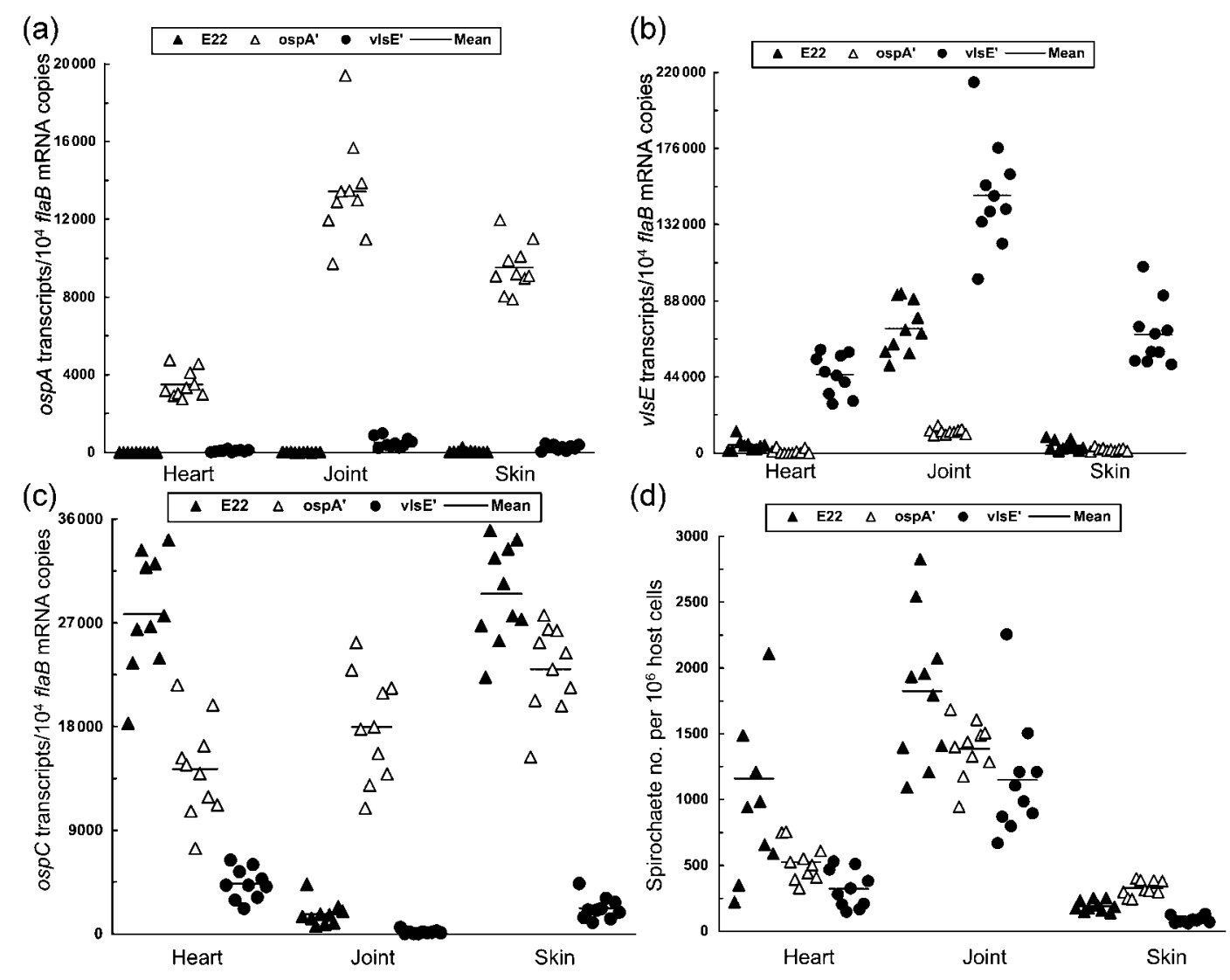

Fig. 2. The influence of overexpression of $\operatorname{osp} A$ and $v / s E$ on gene expression and tissue bacterial loads during infection of SCID mice. Overexpressing $v / s E$ results in increased $\operatorname{osp} A$ (a) but decreased $\operatorname{osp} C$ expression (b), but overexpressing osp $A$ leads to decreased $o s p C$ and $v / s E$ expression (c). Subgroups of five BALB/c SCID mice were inoculated with $10^{4}$ spirochaetes of the clones 13A/E22/C, 13A/E22/D, 13A/ospA'/1, 13A/ospA'/2, 13A/v/sE'/1 and 13A/v/sE'/2. Infected mice were euthanized 1 month later. RNA samples were prepared from heart, joint and skin specimens and analysed for $f l a B$, ospA, ospC and $v / s E$ expression by RT-qPCR. The data are presented as $\operatorname{spp} A(\mathrm{a}), \operatorname{ospC}(\mathrm{b})$ or $v / s E(\mathrm{c}) \mathrm{mRNA}$ copy number per 10000 flaB transcripts and in three groups by combining the subgroups $13 \mathrm{~A} / \mathrm{E} 22 / \mathrm{C}$ and $13 \mathrm{~A} / \mathrm{E} 22 / \mathrm{D}$ (E22), 13A/osp $A^{\prime} / 1$ and $13 \mathrm{~A} /$ $o s p A^{\prime} / 2$ (ospA'), and $13 \mathrm{~A} / v / s E^{\prime} / 1$ and $13 \mathrm{~A} / v / s E^{\prime} / 2$ (vlsE'). (d) Increasing expression of ospA or v/sE leads to a tissuedependent effect on spirochaetal load in SCID mice. DNA samples were prepared from the heart, joint and skin specimens of the 30 mice and analysed for spirochaetal $f l a B$ and murine actin DNA copies by qPCR. The data are expressed as spirochaete numbers per $10^{6}$ host cells and presented in three groups by combining the subgroups $13 \mathrm{~A} / \mathrm{E} 22 / \mathrm{C}$ and $13 \mathrm{~A} / \mathrm{E} 22 / \mathrm{D}, 13 \mathrm{~A} /$ $o s p A^{\prime} / 1$ and $13 \mathrm{~A} / o s p A^{\prime} / 2$, and $13 \mathrm{~A} / v / s E^{\prime} / 1$ and $13 \mathrm{~A} / v / s E^{\prime} / 2$. 
Fig. 2(d), constitutive ospA expression led to a 2.2- and 1.3fold decrease in spirochaete load in heart $(P=0.03)$ and joint tissues $(P=0.03)$, respectively, but a 1.7 -fold increase in skin $\left(P=1.2 \times 10^{-5}\right)$. Increasing $v l s E$ expression resulted in a 3.6-, 1.6-, and 2.2-fold decrease in spirochaete load in heart $(P=0.007)$, joint $(P=0.009)$ and skin tissues $\left(P=1.8 \times 10^{-6}\right)$, respectively. When the two genotypes with increased osp expression were compared, the $13 \mathrm{~A} /$ $o s p A^{\prime}$ spirochaetes generated bacterial loads 1.6- and 3.8fold higher than the $13 \mathrm{~A} / v l s E^{\prime}$ bacteria in heart $(P=0.005)$ and skin tissue $\left(P=1.2 \times 10^{-5}\right)$, but registered similar loads to those of the latter in joint tissue $(P=0.16)$.

\section{Increasing expression of either ospA or vlsE affects dissemination but does not alter ID $_{50}$ in SCID mice}

The influence of increasing ospA or $v l s E$ expression on the $\mathrm{ID}_{50}$ value and dissemination was first investigated in immunodeficient mice. Groups of three animals each received one single inoculation of $10^{1}-10^{3}$ spirochaetes of the clones 13A/E22/C, 13A/E22/D, 13A/ospA'/1, 13A/ $o s p A^{\prime} / 2,13 \mathrm{~A} / v l s E^{\prime} / 1$ and $13 \mathrm{~A} / v l s E^{\prime} / 2$. Ear biopsies were taken for bacterial culture at 2 and 3 weeks postinoculation. Animals were euthanized 1 month postinoculation; heart, joint and skin samples were cultured for $\mathrm{ID}_{50}$ determination. No difference in dissemination was observed between the genotypes $13 \mathrm{~A} / \mathrm{E} 22$ and $13 \mathrm{~A} / \operatorname{os} A^{\prime}$ when the doses were $10^{2}$ or $10^{3}$ organisms, as all inoculated mice in these groups produced a positive ear biopsy at 2 weeks (Supplementary Table S1). However, all five mice that had received 10 organisms of the clones $13 \mathrm{~A} /$ osp $A^{\prime} / 1$ and $13 \mathrm{~A} / \operatorname{sos} p A^{\prime} / 2$ and were found to be infected at the end of the experiments registered a positive biopsy at 2 weeks; in contrast, none of the four infected mice in the same dose group of the clones $13 \mathrm{~A} / \mathrm{E} 22 / \mathrm{C}$ and $13 \mathrm{~A} / \mathrm{E} 22 / \mathrm{D}$ produced a positive biopsy within the time frame, indicating that constitutive ospA expression facilitates dissemination $(P=0.008)$.
When the dose was $10^{3}$ organisms, the $13 \mathrm{~A} / v l s E^{\prime}$ spirochaetes disseminated to and colonized the ear tissue as quickly as genotype 13A/E22, since all inoculated mice in these groups produced a positive ear biopsy at 2 weeks (Supplementary Table S1). When the $10^{2}$ dose groups were compared, the genotype $13 \mathrm{~A} / v l s E^{\prime}$ disseminated at a slower pace than the 13A/E22 spirochaetes, as all the 12 mice that had been inoculated with the clones $13 \mathrm{~A} / \mathrm{E} 22 / \mathrm{C}$ or $13 \mathrm{~A} /$ E22/D in the dose group produced an ear biopsy at 2 weeks, but only two of the 12 mice in the same dose group of the clones $13 \mathrm{~A} / v l_{s} E^{\prime} / 1$ and $13 \mathrm{~A} / v l s E^{\prime} / 2$ gave a positive biopsy within this time frame, indicating that increasing $v l s E$ expression impairs dissemination $\left(P=6.7 \times 10^{-5}\right)$.

The $\mathrm{ID}_{50}$ values for the six clones were measured from six to 18 organisms in repeated experiments (Supplementary Table S1). These results allowed us to conclude that constitutive expression of either ospA or $v l s E$ does not affect the $\mathrm{ID}_{50}$ value in immunodeficient mice $(P>0.05)$.

\section{Increasing the expression of v/sE but not ospA leads to clearance of spirochaetes during early infection of immunocompetent mice}

Subgroups of five $\mathrm{BALB} / \mathrm{c}$ mice each received one single intradermal/subcutaneous injection of $10^{5}$ spirochaetes of the clones $13 \mathrm{~A} / \operatorname{os} p A^{\prime} / 1,13 \mathrm{~A} / \operatorname{sos} p A^{\prime} / 2,13 \mathrm{~A} / v l s E^{\prime} / 1,13 \mathrm{~A} /$ $v l_{s} E^{\prime} / 2,13 \mathrm{~A} / \mathrm{E} 22 / \mathrm{C}$ and $13 \mathrm{~A} / \mathrm{E} 22 / \mathrm{D}$. Animals were euthanized 1 month post-inoculation; heart, joint and skin samples were harvested for bacterial culture and blood samples collected for ELISAs. B. burgdorferi was grown from each specimen of the 10 mice that had received the clones 13A/E22/C and 13A/E22/D (Table 2). Similarly, the $13 \mathrm{~A} / \operatorname{os} p A^{\prime} / 1$ and $13 \mathrm{~A} / \operatorname{os} p A^{\prime} / 2$ spirochaetes were consistently recovered from all the skin samples as well as most of the heart and joint specimens of the 10 inoculated mice. However, no spirochaetes were recovered from the 10 mice that had been inoculated with the clones $13 \mathrm{~A} / v l_{s} E^{\prime} / 1$ and

Table 2. Constitutive expression of $v / s E$ but not $\operatorname{osp} A$ abrogates the ability to infect immunocompetent mice

Groups of five BALB/c mice were inoculated with $10^{5}$ spirochaetes of the clones 13A/E22/C, 13A/E22/D, 13A/ $o s p A^{\prime} / 1,13 \mathrm{~A} / \operatorname{ssp} A^{\prime} / 2,13 \mathrm{~A} / v l s E^{\prime} / 1$ and $13 \mathrm{~A} / v l s E^{\prime} / 2$, and sacrificed 1 month later. Heart, tibiotarsal joint and skin specimens were harvested for spirochaete culture.

\begin{tabular}{|c|c|c|c|c|c|}
\hline \multirow[t]{2}{*}{ Clone } & \multicolumn{4}{|c|}{$\begin{array}{l}\text { Number of cultures positive/total number of specimens } \\
\text { examined }\end{array}$} & \multirow{2}{*}{$\begin{array}{c}\text { Number of mice } \\
\text { infected/ } \\
\text { total number } \\
\text { of mice inoculated }\end{array}$} \\
\hline & Heart & Joint & Skin & All sites & \\
\hline 13A/E22/C & $5 / 5$ & $5 / 5$ & $5 / 5$ & $15 / 15$ & $5 / 5$ \\
\hline 13A/E22/D & $5 / 5$ & $5 / 5$ & $5 / 5$ & $15 / 15$ & $5 / 5$ \\
\hline $13 \mathrm{~A} / \operatorname{osp}^{\prime} A^{\prime} / 1$ & $3 / 5$ & $4 / 5$ & $5 / 5$ & $12 / 15$ & $5 / 5$ \\
\hline $13 \mathrm{~A} / \operatorname{osp} A^{\prime} / 2$ & $4 / 5$ & $4 / 5$ & $5 / 5$ & $13 / 15$ & $5 / 5$ \\
\hline $13 \mathrm{~A} / v l s E^{\prime} / 1$ & $0 / 5$ & $0 / 5$ & $0 / 5$ & $0 / 15$ & $0 / 5$ \\
\hline $13 \mathrm{~A} / v l s E^{\prime} / 2$ & $0 / 5$ & $0 / 5$ & $0 / 5$ & $0 / 15$ & $0 / 5$ \\
\hline
\end{tabular}


$13 \mathrm{~A} / v l s E^{\prime} / 2$, suggesting that increasing $v l s E$ expression abrogates the ability of $B$. burgdorferi to infect immunocompetent mice.

Serum samples collected from the 30 mice were analysed for humoral responses to OspA and VlsE by ELISAs. As shown in Fig. 3(a), infection with the genotype 13A/osp $A^{\prime}$ elicited an anti-OspA response 817-fold stronger than the 13A/E22 spirochaetes $(P=0.003)$. The anti-OspA titre resulting from inoculation with the $13 \mathrm{~A} / v l s E^{\prime}$ spirochaetes was also 7.7-fold higher than that measured in the mice infected with the genotype 13A/E22 $(P=0.02)$. The 13A/E22 spirochaetes elicited an anti-VlsE response 4.5 - and 7.8-fold higher than that of the genotypes $13 \mathrm{~A} / \operatorname{osp}^{\prime}\left(P=1.9 \times 10^{-4}\right)$ and $13 \mathrm{~A} / v l s E^{\prime}\left(P=2.2 \times 10^{-5}\right)$, respectively.

Although the $13 \mathrm{~A} / v l s E^{\prime} / 1$ and $13 \mathrm{~A} / v l s E^{\prime} / 2$ bacteria were not grown from any inoculated mice, the significant humoral responses to OspA and VlsE resulting from inoculation with these clones suggested that the animals might have been infected and subsequently cleared infection as the
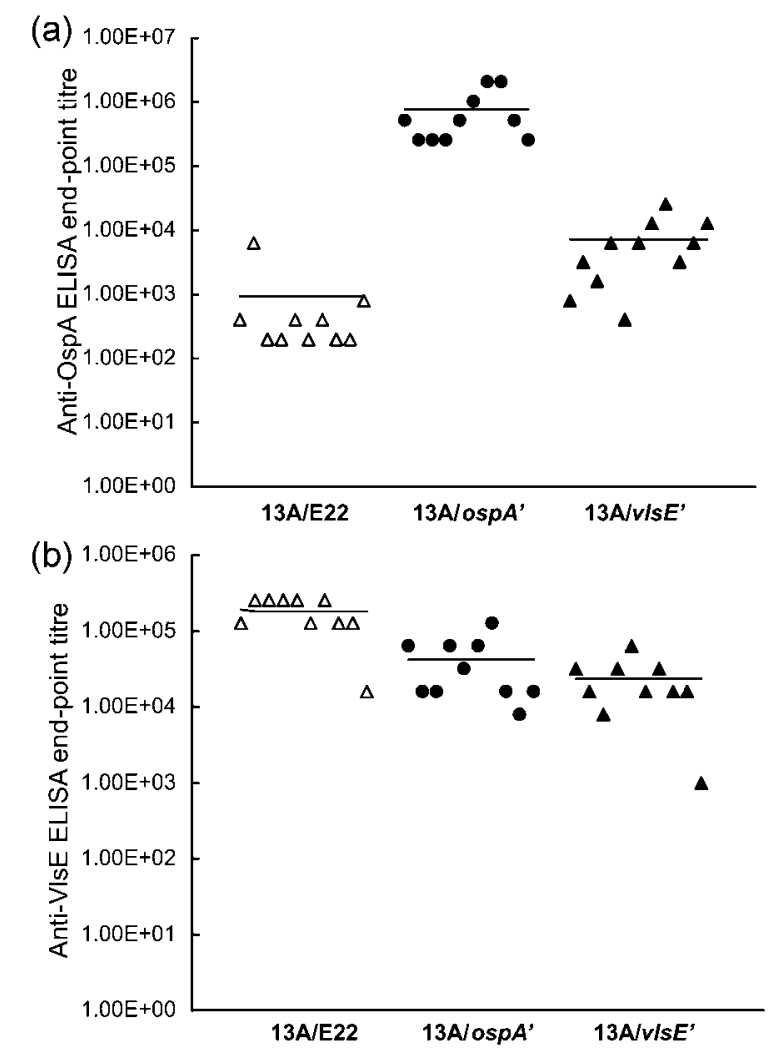

Fig. 3. Humoral responses to OspA (a) and VlsE (b). Subgroups of five BALB/c mice were inoculated with the clones 13A/E22/C, 13A/E22/D, 13A/ospA'/1, 13A/ospA'/2, 13A/v/sE'/1 and 13A/ $v / s E^{\prime} / 2$, and euthanized 1 month later. Serum samples were analysed for anti-OspA and -VlsE antibody titres by end-point ELISAs. The data are presented in three groups by combining the subgroups 13A/E22/C and 13A/E22/D, 13A/ospA'/1 and 13A/ $o s p A^{\prime} / 2$, and $13 \mathrm{~A} / v / s E^{\prime} / 1$ and $13 \mathrm{~A} / \mathrm{v} / s E^{\prime} / 2$. specific immune responses developed. To clarify the issue, groups of $12 \mathrm{BALB} / \mathrm{c}$ mice each received a single intradermal/subcutaneous inoculation of $10^{5}$ spirochaetes of the clones 13A/E22/C, 13A/E22/D, 13A/ospA'/1, 13A/ $o s p A^{\prime} / 2,13 \mathrm{~A} / v l s E^{\prime} / 1$ and $13 \mathrm{~A} / v l s E^{\prime} / 2$. Three animals from each group were euthanized at 1-week intervals; the inoculation site and remote skin, ear, heart and joint specimens were harvested for spirochaete isolation. As a positive control, the 13A/E22/C and 13A/E22/D bacteria disseminated quickly, and colonized all tissues within 2 weeks (Table 3). The 13A/osp $A^{\prime} / 1$ and 13A/osp $A^{\prime} / 2$ spirochaetes disseminated slowly, and all ear tissue cultures did not become positive until 4 weeks post-inoculation, although all joint samples were positive within the first week. The $13 \mathrm{~A} / v l s E^{\prime} / 1$ and $13 \mathrm{~A} / v l s E^{\prime} / 2$ bacteria were recovered from at least one tissue of each inoculated mouse within the first 3 weeks, but none of the tissues was positive at 4 weeks post-inoculation, indicating that $B$. burgdorferi with increased $v l s E$ expression can initiate infection but is subsequently eliminated.

\section{Constitutive ospA expression dramatically increases ID $_{50}$ and severely impairs dissemination in immunocompetent mice}

The influence of constitutive ospA expression on the $\mathrm{ID}_{50}$ value and dissemination was investigated in immunocompetent mice. In experiment I, groups of three animals each received one single inoculation of $10^{1}-10^{4}$ spirochaetes of the clones 13A/E22/C, 13A/E22/D, 13A/osp $A^{\prime} / 1$ and $13 \mathrm{~A} /$ $o s p A^{\prime} / 2$. Ear biopsies were taken for bacterial culture at 2, 3,4 and 5 weeks post-inoculation. Animals were euthanized 6 weeks post-inoculation; heart, joint and skin samples were cultured for spirochaetes. At 2 weeks, almost all mice that had been challenged with $10^{2}$ or more organisms of the clones $13 \mathrm{~A} / \mathrm{E} 22 / \mathrm{C}$ and $13 \mathrm{~A} / \mathrm{E} 22 / \mathrm{D}$ produced a positive biopsy; the remaining mice that were found to be infected at the end of the experiment became positive a week later (Supplementary Table S2). In contrast, none of the mice inoculated with the clones $13 \mathrm{~A} /$ osp $p A^{\prime} / 1$ and $13 \mathrm{~A} / \operatorname{osp} A^{\prime} / 2$ produced a positive biopsy at 2 weeks; most of the mice that were found to be infected at the end of the experiment did not develop a positive ear biopsy until week 4 . The study allowed us to conclude that increasing ospA expression severely delays dissemination during infection of immunocompetent mice.

The $\mathrm{ID}_{50}$ values of the clones $13 \mathrm{~A} / \mathrm{E} 22 / \mathrm{C}$ and $13 \mathrm{~A} / \mathrm{E} 22 / \mathrm{D}$ were 18 and 32 organisms, compared to 178 and 56 for the clones $13 \mathrm{~A} / \operatorname{os} p A^{\prime} / 1$ and $13 \mathrm{~A} / \operatorname{osp} A^{\prime} / 2$ in experiment I (Supplementary Table S2). The values were 18 and 32 for the clones $13 \mathrm{~A} / \mathrm{E} 22 / \mathrm{C}$ and $13 \mathrm{~A} / \mathrm{E} 22 / \mathrm{D}$, and 178 and 178 for the clones $13 \mathrm{~A} / \operatorname{os} p A^{\prime} / 1$ and $13 \mathrm{~A} / \operatorname{osp} A^{\prime} / 2$, respectively, in experiment II. Taken together, the study indicated that increasing ospA expression results in a 24 -fold increase in $\mathrm{ID}_{50}$ in immunocompetent mice $(P=0.007)$.

Another defect resulting from constitutive ospA expression was a reduced frequency in colonizing heart and joint 
Table 3. B. burgdorferi with constitutive $v / s E$ expression is cleared during early infection of immunocompetent mice

Groups of $12 \mathrm{BALB} / \mathrm{c}$ mice each received a single intradermal/subcutaneous injection of $10^{5}$ organisms of the clones 13A/E22/C, 13A/E22/D, 13A/ $\operatorname{osp} A^{\prime} / 1,13 \mathrm{~A} / \operatorname{sp} A^{\prime} / 2,13 \mathrm{~A} / v l s E^{\prime} / 1$ and $13 \mathrm{~A} / v l s E^{\prime} / 2$. Three animals from each group were euthanized at $1,2,3$ and 4 weeks post-inoculation; inoculation site (I.S.) and remote site (R.S.) skin, ear, heart and joint specimens were harvested for spirochaete isolation. The I.S. site was at the chest; therefore, the R.S. site was at the back of mice.

\begin{tabular}{|c|c|c|c|c|c|c|c|c|c|c|c|c|c|c|c|c|c|c|c|c|}
\hline \multirow[t]{3}{*}{ Clone } & \multicolumn{20}{|c|}{ Number of specimens positive/total number of specimens examined at post-inoculation week: } \\
\hline & \multicolumn{5}{|c|}{1} & \multicolumn{5}{|c|}{2} & \multicolumn{5}{|c|}{3} & \multicolumn{5}{|c|}{4} \\
\hline & I.S. & R.S. & Ear & Heart & Joint & I.S. & R.S. & Ear & Heart & Joint & I.S. & R.S. & Ear & Heart & Joint & I.S. & R.S. & Ear & Heart & Joint \\
\hline 13A/E22/C & $3 / 3$ & $1 / 3$ & $0 / 3$ & $0 / 3$ & $3 / 3$ & $3 / 3$ & $3 / 3$ & $3 / 3$ & $3 / 3$ & $3 / 3$ & $3 / 3$ & $3 / 3$ & $3 / 3$ & $3 / 3$ & $3 / 3$ & $3 / 3$ & $3 / 3$ & $3 / 3$ & $3 / 3$ & $3 / 3$ \\
\hline 13A/E22/D & $3 / 3$ & $0 / 3$ & $0 / 3$ & $0 / 3$ & $3 / 3$ & $3 / 3$ & $3 / 3$ & $3 / 3$ & $3 / 3$ & $3 / 3$ & $3 / 3$ & $3 / 3$ & $3 / 3$ & $3 / 3$ & $3 / 3$ & $3 / 3$ & $3 / 3$ & $3 / 3$ & $3 / 3$ & $3 / 3$ \\
\hline $13 \mathrm{~A} / \operatorname{osp} A^{\prime} / 1$ & $3 / 3$ & $0 / 3$ & $0 / 3$ & $0 / 3$ & $3 / 3$ & $0 / 3$ & $3 / 3$ & $0 / 3$ & $2 / 3$ & $3 / 3$ & $3 / 3$ & $3 / 3$ & $2 / 3$ & $2 / 3$ & $3 / 3$ & $3 / 3$ & $3 / 3$ & $3 / 3$ & $2 / 3$ & $2 / 3$ \\
\hline $13 \mathrm{~A} / \operatorname{spp} A^{\prime} / 2$ & $3 / 3$ & $1 / 3$ & $0 / 3$ & $0 / 3$ & $3 / 3$ & $0 / 3$ & $3 / 3$ & $0 / 3$ & $3 / 3$ & $3 / 3$ & $3 / 3$ & $3 / 3$ & $3 / 3$ & $2 / 3$ & $2 / 3$ & $3 / 3$ & $3 / 3$ & $3 / 3$ & $2 / 3$ & $3 / 3$ \\
\hline $13 \mathrm{~A} / v l s E^{\prime} / 1$ & $0 / 3$ & $0 / 3$ & $0 / 3$ & $0 / 3$ & $3 / 3$ & $0 / 3$ & $1 / 3$ & $1 / 3$ & $0 / 3$ & $2 / 3$ & $0 / 3$ & $1 / 3$ & $1 / 3$ & $0 / 3$ & $1 / 3$ & $0 / 3$ & $0 / 3$ & $0 / 3$ & $0 / 3$ & $0 / 3$ \\
\hline $13 \mathrm{~A} / v l s E^{\prime} / 2$ & $0 / 3$ & $0 / 3$ & $0 / 3$ & $0 / 3$ & $3 / 3$ & $0 / 3$ & $2 / 3$ & $1 / 3$ & $0 / 3$ & $2 / 3$ & $0 / 3$ & $1 / 3$ & $0 / 3$ & $1 / 3$ & $1 / 3$ & $0 / 3$ & $0 / 3$ & $0 / 3$ & $0 / 3$ & $0 / 3$ \\
\hline
\end{tabular}

tissues. The $13 \mathrm{~A} / \operatorname{sos} p A^{\prime} / 1$ and $13 \mathrm{~A} / \operatorname{sos} A^{\prime} / 2$ spirochaetes were grown from only 10 heart and 15 joint specimens of the 23 infected mice; in contrast, the clones $13 \mathrm{~A} / \mathrm{E} 22 / \mathrm{C}$ and $13 \mathrm{~A} / \mathrm{E} 22 / \mathrm{D}$ were recovered from each specimen from all of the 26 infected mice (Supplementary Table S2). Overall, constitutive ospA expression led to a 67 and $35 \%$ decrease in frequency of colonizing heart $\left(P=4.4 \times 10^{-6}\right)$ and joint tissues $\left(P=1.1 \times 10^{-3}\right)$, respectively.

\section{DISCUSSION}

In the current study, B. burgdorferi was modified to increase expression of two well-characterized surface lipoproteins, OspA and VlsE, leading to dramatic changes in infectious behaviour. Increasing the expression of either ospA or $v l s E$ did not alter the $\mathrm{ID}_{50}$ value, but affected spirochaetal dissemination and significantly reduced tissue spirochaete loads in SCID mice. In immunocompetent mice, increased $v l s E$ expression resulted in quick clearance of infection, while constitutive ospA expression led to a substantial $\mathrm{ID}_{50}$ increase and severely impaired dissemination. The study also showed that $B$. burgdorfer $i$ with constitutive ospA expression persisted in skin tissue but was cleared from both heart and joints of chronically infected immunocompetent mice. Overall, the study highlights a relationship between the surface antigen expression and the infectious behaviour of $B$. burgdorferi.

Both $\operatorname{ssp} A$ and $v l s E$ use $\sigma^{70}$-dependent promoters, while the ospC promoter is RpoS-dependent (Hubner et al., 2001). Within mammals, however, ospA expression is essentially repressed, while $v l s E$ expression is upregulated, especially during chronic infection of immunocompetent hosts (Liang et al., 2004b). Nothing is known about how this could occur. Nevertheless, our RT-qPCR results showed that constitutive ospA expression repressed $v l s E$ and $o s p C$ expression, and that increased $v l s E$ expression upregulated ospA but downregulated ospC in infected SCID mice. The same scenario also appeared to occur in infected immunocompetent mice, as $B$. burgdorferi with constitutive ospA expression elicited a weaker anti-VlsE response, but bacteria with increased $v l s E$ expression stimulated a stronger anti-OspA response than

Table 4. B. burgdorferi with increased $\operatorname{ssp} A$ expression persists only in the skin tissue of chronically infected immunocompetent mice

Groups of five BALB/c mice were inoculated with the clones 13A/E22/C, 13A/E22/D, 13A/osp $A^{\prime} / 1$ and 13A/osp $A^{\prime} / 2$, and sacrificed 4 months later. Heart, tibiotarsal joint and skin specimens were harvested for spirochaete culture.

\begin{tabular}{|c|c|c|c|c|c|}
\hline \multirow[t]{2}{*}{ Clone } & \multicolumn{4}{|c|}{$\begin{array}{c}\text { Number of cultures positive/total number of specimens } \\
\text { examined }\end{array}$} & \multirow{2}{*}{$\begin{array}{l}\text { Number of mice } \\
\text { infected/ } \\
\text { total number of } \\
\text { mice inoculated }\end{array}$} \\
\hline & Heart & Joint & Skin & All sites & \\
\hline 13A/E22/C & $5 / 5$ & $4 / 5$ & $5 / 5$ & $14 / 15$ & $5 / 5$ \\
\hline 13A/E22/D & $5 / 5$ & $5 / 5$ & $5 / 5$ & $15 / 15$ & $5 / 5$ \\
\hline $13 \mathrm{~A} / \cos p A^{\prime} / 1$ & $0 / 5$ & $0 / 5$ & $5 / 5$ & $5 / 15$ & $5 / 5$ \\
\hline $13 \mathrm{~A} / \operatorname{osp}^{\prime} A^{\prime} / 2$ & $0 / 5$ & $0 / 5$ & $5 / 5$ & $5 / 15$ & $5 / 5$ \\
\hline
\end{tabular}


the control. It would be interesting to address how an increase in lipoprotein gene expression can influence the expression of other lipoproteins.

To initiate an infection, B. burgdorferi must be able to evade innate immunity and colonize local tissues of the inoculation site; this ability was measured by $\mathrm{ID}_{50}$ values in immunodeficient mice. Apparently, increasing ospA or $v l s E$ expression did not affect this ability, as increasing the expression of either did not change the value in SCID mice. However, increasing $v l s E$ expression slightly impaired dissemination, and constitutive ospA expression apparently facilitated the process in the absence of adaptive immunity, underscoring the importance of surface antigen expression in spirochaetal dissemination. Our previous study showed that increasing expression of DbpA severely impairs dissemination (Xu et al., 2007b). Even more dramatically, overproducing DbpA restrains OspC-deficient B. burgdorferi to tissues at the inoculation site for 4 weeks without dissemination to distal tissues, further highlighting the influence of surface lipoprotein expression on dissemination ( $\mathrm{Xu}$ et al., 2008). OspC may function as a dissemination-facilitating factor (Xu et al., 2008), and has been found to bind plasminogen (Lagal et al., 2006). Interestingly, OspA is a primary plasmin(ogen) receptor (Fuchs et al., 1994), and in vitro evidence shows that OspA helps B. burgdorferi to acquire plasmin(ogen) (Coleman et al., 1995; Hu et al., 1995; Klempner et al., 1996). Plasmin, a trypsin-like serine proteinase, is generated by limited proteolysis from its serum-derived zymogen precursor plasminogen by the urokinase-type plasminogen activator or tissue-type plasminogen activator. An in vitro study has shown that B. burgdorferi coated with plasmin gains a proteolytic activity to effectively degrade components of the mammalian extracellular matrix (Coleman et al., 1999), and an animal study has indicated that plasminogen is required for efficient dissemination of B. burgdorferi in ticks and for enhancement of spirochaetaemia in mice (Coleman et al., 1997). It remains to be addressed whether facilitated dissemination due to constitutive ospA expression results from gaining host plasminogen.

As an extracellular pathogen, B. burgdorferi resides primarily in the extracellular matrix and connective tissues as well as between host cells during mammalian infection. The influence of surface antigen expression on this aspect was reflected by tissue bacterial load. Increasing expression of either $\operatorname{ssp} A$ or $v l s E$ negatively affected the ability of $B$. burgdorferi to colonize most tissues. This adverse effect of high $v l s E$ expression underscores the biological significance of low expression during early infection. B. burgdorferi does not actively express $v l s E$ but increases expression after dissemination into joint tissues in the absence of adaptive immune responses (Crother et al., 2003; Liang et al., 2004b). Low $v l s E$ expression may also allow bacteria to more efficiently disseminate to distal tissues, as the current study showed that increasing $v l s E$ expression led to slow dissemination in SCID mice. After dissemination, humoral responses greatly upregulate $v l s E$ in all tissues (Crother $e t$ al., 2004; Liang et al., 2004b), an event consistent with the critical role of VlsE in immune evasion during chronic infection of immunocompetent hosts (Bankhead \& Chaconas, 2007; Zhang et al., 1997).

Naive and immune statuses constitute two distinct environments to microbial pathogens. The current study used immunodeficient and immunocompetent mice to provide these environments in order to study the influence of increased surface lipoprotein expression on infectious behaviour. Increasing the expression of $o s p A$ or $v l s E$ did not affect the $\mathrm{ID}_{50}$ value in SCID mice. In sharp contrast, increased $v l s E$ expression diminished the ability to infect immunocompetent mice. Although a less significant effect attributed to increased ospA expression was observed in immunocompetent mice, $B$. burgdorferi with constitutive ospA expression registered a substantial $\mathrm{ID}_{50}$ increase and disseminated at a much slower pace. Even more dramatically, constitutive ospA expression diminished the ability to persist in both heart and joint tissues during chronic infection of immunocompetent mice.

An earlier study has shown that simultaneous constitutive expression of OspA and OspB abrogates infectivity in immunocompetent mice (Strother et al., 2007). In the current study, although constitutive ospA expression significantly increased the $\mathrm{ID}_{50}$ value and severely impaired dissemination, recombinant spirochaetes were able to persist in the skin tissue of chronically infected immunocompetent mice. Co-expressing OspA and OspB gives the immune system two targets, so that recombinant spirochaetes may be quickly cleared by the humoral responses after inoculation into immunocompetent mice. Alternatively, OspB is more effectively targeted than OspA by protective immunity, so that the anti-OspB response alone is sufficient to clear infection.

Increasing the expression of a lipoprotein appears to be an efficient way to test whether the specific antigen is effectively targeted by protective immunity. To date, four surface lipoproteins, OspC, DbpA, OspA and VlsE, have been individually examined in this way (Xu et al., 2006, 2007b). B. burgdorferi modified with increased expression of $d b p A$ or $\operatorname{csp} A$ can persist in the skin tissue of chronically infected immunocompetent mice (Xu et al., 2007b). Although constitutive ospC expression diminishes the ability to cause persistent infection, recombinant spirochaetes are consistently recovered from immunocompetent mice for at least a month after infection (Xu et al., 2006). In contrast, $B$. burgdorferi with constitutive invariant $v l s E$ expression is cleared within a month after inoculation into immunocompetent mice, although the production of invariant VlsE antigen under the control of its own promoter allows spirochaetes to cause persistent infection (Lawrenz et al., 2004). Taken together, these studies indicate that VlsE, when no antigenic variation occurs, is the most effective target of protective immunity.

B. burgdorferi actively modifies its surface antigen expression in order to better adapt to the tissue microenvironment 
during the course of mammalian infection. The current study shows that artificially increasing surface antigen expression alters infectious behaviour in a negative way, highlighting the importance of the coordination of surface antigen expression in the pathogenesis of B. burgdorferi.

\section{ACKNOWLEDGEMENTS}

This work was in part supported by a career development award and a grant from NIH/NIAMS, an Arthritis Foundation Investigators award, and P20RR020159 (PI, Kousoulas) from NIH/NCRR.

\section{REFERENCES}

Bankhead, T. \& Chaconas, G. (2007). The role of VlsE antigenic variation in the Lyme disease spirochete: persistence through a mechanism that differs from other pathogens. Mol Microbiol 65, 1547-1558.

Bykowski, T., Woodman, M. E., Cooley, A. E., Brissette, C. A., Brade, V., Wallich, R., Kraiczy, P. \& Stevenson, B. (2007). Coordinated expression of Borrelia burgdorferi complement regulator-acquiring surface proteins during the Lyme disease spirochete's mammal-tick infection cycle. Infect Immun 75, 4227-4236.

Coleman, J. L., Sellati, T. J., Testa, J. E., Kew, R. R., Furie, M. B. \& Benach, J. L. (1995). Borrelia burgdorferi binds plasminogen, resulting in enhanced penetration of endothelial monolayers. Infect Immun 63, 2478-2484.

Coleman, J. L., Gebbia, J. A., Piesman, J., Degen, J. L., Bugge, T. H. \& Benach, J. L. (1997). Plasminogen is required for efficient dissemination of $B$. burgdorferi in ticks and for enhancement of spirochetemia in mice. Cell 89, 1111-1119.

Coleman, J. L., Roemer, E. J. \& Benach, J. L. (1999). Plasmin-coated Borrelia burgdorferi degrades soluble and insoluble components of the mammalian extracellular matrix. Infect Immun 67, 3929-3936.

Crother, T. R., Champion, C. I., Wu, X. Y., Blanco, D. R., Miller, J. N. \& Lovett, M. A. (2003). Antigenic composition of Borrelia burgdorferi during infection of SCID mice. Infect Immun 71, 3419-3428.

Crother, T. R., Champion, C. I., Whitelegge, J. P., Aguilera, R., Wu, X. Y., Blanco, D. R., Miller, J. N. \& Lovett, M. A. (2004). Temporal analysis of the antigenic composition of Borrelia burgdorferi during infection in rabbit skin. Infect Immun 72, 5063-5072.

de Silva, A. M., Telford, S. R., III, Brunet, L. R., Barthold, S. W. \& Fikrig, E. (1996). Borrelia burgdorferi OspA is an arthropod-specific transmission-blocking Lyme disease vaccine. J Exp Med 183, 271-275.

de Silva, A. M., Fish, D., Burkot, T. R., Zhang, Y. \& Fikrig, E. (1997). OspA antibodies inhibit the acquisition of Borrelia burgdorferi by Ixodes ticks. Infect Immun 65, 3146-3150.

Fingerle, V., Goettner, G., Gern, L., Wilske, B. \& Schulte-Spechtel, U. (2007). Complementation of a Borrelia afzelii OspC mutant highlights the crucial role of OspC for dissemination of Borrelia afzelii in Ixodes ricinus. Int J Med Microbiol 297, 97-107.

Fuchs, H., Wallich, R., Simon, M. M. \& Kramer, M. D. (1994). The outer surface protein A of the spirochete Borrelia burgdorferi is a plasmin(ogen) receptor. Proc Natl Acad Sci U S A 91, 12594-12598.

Fung, B. P., McHugh, G. L., Leong, J. M. \& Steere, A. C. (1994). Humoral immune response to outer surface protein $\mathrm{C}$ of Borrelia burgdorferi in Lyme disease: role of the immunoglobulin $\mathrm{M}$ response in the serodiagnosis of early infection. Infect Immun 62, 3213-3221.

Gilmore, R. D., Jr \& Piesman, J. (2000). Inhibition of Borrelia burgdorferi migration from the midgut to the salivary glands following feeding by ticks on OspC-immunized mice. Infect Immun 68, 411-414.

Gilmore, R. D., Jr, Howison, R. R., Schmit, V. L., Nowalk, A. J., Clifton, D. R., Nolder, C., Hughes, J. L. \& Carroll, J. A. (2007). Temporal expression analysis of the Borrelia burgdorferi paralogous gene family 54 genes BBA64, BBA65, and BBA66 during persistent infection in mice. Infect Immun 75, 2753-2764.

Grimm, D., Tilly, K., Byram, R., Stewart, P. E., Krum, J. G., Bueschel, D. M., Schwan, T. G., Policastro, P. F., Elias, A. F. \& Rosa, P. A. (2004). Outer-surface protein $C$ of the Lyme disease spirochete: a protein induced in ticks for infection of mammals. Proc Natl Acad Sci U S A 101, 3142-3147.

Hu, L. T., Perides, G., Noring, R. \& Klempner, M. S. (1995). Binding of human plasminogen to Borrelia burgdorferi. Infect Immun 63, 3491-3496.

Hubner, A., Yang, X., Nolen, D. M., Popova, T. G., Cabello, F. C. \& Norgard, M. V. (2001). Expression of Borrelia burgdorferi OspC and DbpA is controlled by a RpoN-RpoS regulatory pathway. Proc Natl Acad Sci U S A 98, 12724-12729.

Klempner, M. S., Noring, R., Epstein, M. P., McCloud, B. \& Rogers, R. A. (1996). Binding of human urokinase type plasminogen activator and plasminogen to Borrelia species. J Infect Dis 174, 97-104.

Lagal, V., Portnoi, D., Faure, G., Postic, D. \& Baranton, G. (2006). Borrelia burgdorferi sensu stricto invasiveness is correlated with OspCplasminogen affinity. Microbes Infect 8, 645-652.

Lawrenz, M. B., Wooten, R. M. \& Norris, S. J. (2004). Effects of $v l s E$ complementation on the infectivity of Borrelia burgdorferi lacking the linear plasmid lp28-1. Infect Immun 72, 6577-6585.

Liang, F. T., Jacobs, M. B., Bowers, L. C. \& Philipp, M. T. (2002a). An immune evasion mechanism for spirochetal persistence in Lyme borreliosis. J Exp Med 195, 415-422.

Liang, F. T., Nelson, F. K. \& Fikrig, E. (2002b). Molecular adaptation of Borrelia burgdorferi in the murine host. J Exp Med 196, 275-280.

Liang, F. T., Caimano, M. J., Radolf, J. D. \& Fikrig, E. (2004a). Borrelia burgdorferi outer surface protein (osp) B expression independent of ospA. Microb Pathog 37, 35-40.

Liang, F. T., Yan, J., Mbow, M. L., Sviat, S. L., Gilmore, R. D., Mamula, M. \& Fikrig, E. (2004b). Borrelia burgdorferi changes its surface antigenic expression in response to host immune responses. Infect Immun 72, 5759-5767.

Neelakanta, G., Li, X., Pal, U., Liu, X., Beck, D. S., Deponte, K., Fish, D., Kantor, F. S. \& Fikrig, E. (2007). Outer surface protein B is critical for Borrelia burgdorferi adherence and survival within Ixodes ticks. PLoS Pathog 3, e33.

Ohnishi, J., Piesman, J. \& de Silva, A. M. (2001). Antigenic and genetic heterogeneity of Borrelia burgdorferi populations transmitted by ticks. Proc Natl Acad Sci U S A 98, 670-675.

Pal, U., Yang, X., Chen, M., Bockenstedt, L. K., Anderson, J. F., Flavell, R. A., Norgard, M. V. \& Fikrig, E. (2004). OspC facilitates Borrelia burgdorferi invasion of Ixodes scapularis salivary glands. J Clin Invest 113, 220-230.

Purser, J. E., Lawrenz, M. B., Caimano, M. J., Howell, J. K., Radolf, J. D. \& Norris, S. J. (2003). A plasmid-encoded nicotinamidase (PncA) is essential for infectivity of Borrelia burgdorferi in a mammalian host. Mol Microbiol 48, 753-764.

Schwan, T. G. \& Piesman, J. (2000). Temporal changes in outer surface proteins A and C of the Lyme disease-associated spirochete, Borrelia burgdorferi, during the chain of infection in ticks and mice. J Clin Microbiol 38, 382-388.

Schwan, T. G., Piesman, J., Golde, W. T., Dolan, M. C. \& Rosa, P. A. (1995). Induction of an outer surface protein on Borrelia burgdorferi during tick feeding. Proc Natl Acad Sci U S A 92, 2909-2913. 
Stewart, P. E., Wang, X., Bueschel, D. M., Clifton, D. R., Grimm, D., Tilly, K., Carroll, J. A., Weis, J. J. \& Rosa, P. A. (2006). Delineating the requirement for the Borrelia burgdorferi virulence factor OspC in the mammalian host. Infect Immun 74, 3547-3553.

Strother, K. O., Hodzic, E., Barthold, S. W. \& de Silva, A. M. (2007). Infection of mice with Lyme disease spirochetes constitutively producing outer surface proteins A and B. Infect Immun 75, 2786-2794.

Tilly, K., Krum, J. G., Bestor, A., Jewett, M. W., Grimm, D., Bueschel, D., Byram, R., Dorward, D., Vanraden, M. J. \& other authors (2006). Borrelia burgdorferi OspC protein required exclusively in a crucial early stage of mammalian infection. Infect Immun 74, 3554-3564.

Tsao, J., Barbour, A. G., Luke, C. J., Fikrig, E. \& Fish, D. (2001). OspA immunization decreases transmission of Borrelia burgdorferi spirochetes from infected Peromyscus leucopus mice to larval Ixodes scapularis ticks. Vector Borne Zoonotic Dis 1, 65-74.

Tsao, J. I., Wootton, J. T., Bunikis, J., Luna, M. G., Fish, D. \& Barbour, A. G. (2004). An ecological approach to preventing human infection: vaccinating wild mouse reservoirs intervenes in the Lyme disease cycle. Proc Natl Acad Sci U S A 101, 18159-18164.

Xu, Q., Seemanapalli, S. V., Lomax, L., McShan, K., Li, X., Fikrig, E. \& Liang, F. T. (2005). Association of linear plasmid 28-1 with an arthritic phenotype of Borrelia burgdorferi. Infect Immun 73, 7208-7215.
Xu, Q., Seemanapalli, S. V., McShan, K. \& Liang, F. T. (2006). Constitutive expression of outer surface protein $C$ diminishes the ability of Borrelia burgdorferi to evade specific humoral immunity. Infect Immun 74, 5177-5184.

Xu, Q., McShan, K. \& Liang, F. T. (2007a). Identification of an ospC operator critical for immune evasion of Borrelia burgdorferi. Mol Microbiol 64, 220-231.

Xu, Q., Seemanaplli, S. V., McShan, K. \& Liang, F. T. (2007b). Increasing the interaction of Borrelia burgdorferi with decorin significantly reduces the 50 percent infectious dose and severely impairs dissemination. Infect Immun 75, 4272-4281.

Xu, Q., McShan, K. \& Liang, F. T. (2008). Essential protective role attributed to the surface lipoproteins of Borrelia burgdorferi against innate defences. Mol Microbiol 69, 15-29.

Yang, X. F., Pal, U., Alani, S. M., Fikrig, E. \& Norgard, M. V. (2004). Essential role for OspA/B in the life cycle of the Lyme disease spirochete. J Exp Med 199, 641-648.

Zhang, J. R., Hardham, J. M., Barbour, A. G. \& Norris, S. J. (1997). Antigenic variation in Lyme disease borreliae by promiscuous recombination of VMP-like sequence cassettes. Cell 89, 275-285.

Edited by: G. E. Duhamel 